\title{
Incidental Enterogenous Cyst of the Pancreas: Resection or Observation?
}

\author{
Lauren Smithson, MD, Michael J. Jacobs, MD \\ Department of Surgery, Providence Hospital and Medical Centers, Southfield, MI, USA (both authors).
}

\begin{abstract}
Introduction: Enterogenous cysts of the pancreas are rare congenital foregut cysts. The natural history of these entities is still relatively unknown, as is their propensity for malignant transformation. Thus, optimal management is still undetermined.

Case Description: A 39-year-old African American woman underwent a computed tomography scan of the chest that showed an incidental cystic lesion of the pancreas. Endoscopic ultrasonography-guided fine-needle aspiration (FNA) identified an elevated carcinoembryonic antigen level, as well as benign, focally ciliated columnar cells, histiocytes, and proteinaceous material, with no evidence of malignant transformation. These findings were consistent with respiratory or bronchial versus dermoid or endometrial (müllerian) origin. A laparoscopic distal pancreatectomy was performed, and the final pathologic findings showed gastric tissue surrounded by a rim of normal pancreatic tissue, consistent with a benign enterogenous cyst. The patient recovered well.

Discussion: Enterogenous cysts that arise outside of the liver are exceedingly rare and represent both a diagnostic and therapeutic challenge. On imaging, they can be mistaken for malignant cystic lesions. Pathologic examination provides definitive identification, with FNA smears giving pathognomonic characteristics; however, FNA samples can miss the architecture of the cyst. Moreover, there is limited knowledge of the natural history of these lesions, and reports exist of other enterogenous or developmental abnormalities undergoing malignant transformation. Although it would be preferential to avoid unnecessary surgery for these rare lesions, not enough is known about their behavior. Surgery remains the standard of care and the more "conservative" management option for these cystic lesions of the pancreas.
\end{abstract}

Key Words: Enterogenous cyst, Pancreas, Laparoscopic distal pancreatectomy

Citation Smithson L, Jacobs MJ. Incidental enterogenous cyst of the pancreas: resection or observation? CRSLS e2015.00053. DOI: 10.4293/CRSLS.2015.00053.

Copyright (c) 2015 by SLS, Society of Laparoendoscopic Surgeons. This is an open-access article distributed under the terms of the Creative Commons Attribution-Noncommercial-ShareAlike 3.0 Unported license, which permits unrestricted noncommercial use, distribution, and reproduction in any medium, provided the original author and source are credited.

The authors acknowledge Marc Zuckman, MD, Department of Pathology, Providence Hospital and Medical Centers, Southfield, MI, USA.

Address correspondence to: Lauren Smithson, MD, Department of General Surgery, Providence Hospital and Medical Centers, 16001 W Nine Mile Rd, Southfield, MI 48075, USA. Telephone: (248) 849-3073, extension 2, Fax: (248) 849-5380, E-mail address: lauren_smithson@yahoo.com

\section{INTRODUCTION}

Enterogenous cysts are typically congenital foregut cysts rarely found in the subdiaphragmatic area. Even more rarely are these enterogenous cysts found in the pancreas. Since the first reported case of an enterogenous cyst of the ampulla of Vater in 1969, only 14 cases of pancreatic ciliated foregut cysts have been mentioned in the literature. ${ }^{1-3}$ These cysts form as abnormal developments of the foregut during the fourth week of gestation. ${ }^{4}$ They will either remain attached to the foregut or branch off. The ventral bud of the foregut forms the respiratory tract, and the dorsal bud forms the esophagus, stomach, liver, pancreas, and biliary tree. Histologically, most cysts are lined with pseudostratified ciliated epithelium, but they can contain foci of enteric tissue. ${ }^{3}$ Cysts typically contain serous or proteinaceous milky fluid, and they can have elevated carcinoembryonic antigen (CEA) and cancer antigen (CA) 19-9 levels. 4,5 The natural history of these entities is still relatively unknown, as is their propensity for malignant transformation. Thus, optimal management is still undetermined. Balancing the risks of surgery versus the safety of observation becomes the central question.

\section{CASE PRESENTATION}

A 39-year-old African American woman presented to the emergency department with chest pain. A com- 
Incidental Enterogenous Cyst of the Pancreas, Smithson L et al.

A

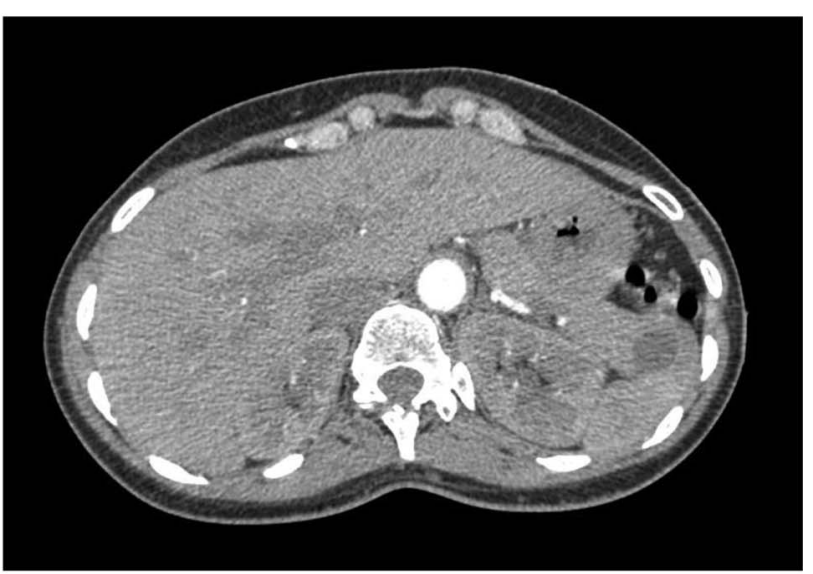

B

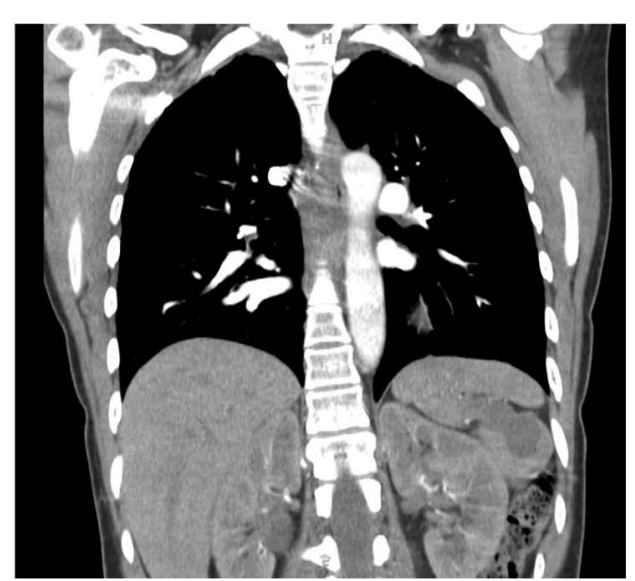

Figure 1. Computed tomography scans of incidental cystic pancreatic tail mass: axial view (A) and coronal view (B).

puted tomography scan of the chest showed an incidental pancreatic tail lesion (Figure 1). A magnetic resonance imaging scan was subsequently performed, showing a non-enhancing lobulated cystic lesion of the pancreatic tail suggestive of a mucinous cystic pancreatic neoplasm (Figure 2). Endoscopic ultrasonography (EUS) showed a unilocular cyst measuring $4 \times 2.8 \mathrm{~cm}$ with cloudy beige aspirate. On pathologic analysis, the smear contained benign, focally ciliated columnar cells, histiocytes, and proteinaceous material, with no evidence of malignant transformation. These findings were consistent with respiratory or bronchial versus dermoid or endometrial (müllerian) origin. The CEA level of the aspirate was 5450 $\mathrm{ng} / \mathrm{mL}$. The serum CEA level was $0.7 \mathrm{ng} / \mathrm{mL}$, the CA $19-9$ level was $1.0 \mathrm{U} / \mathrm{mL}$, and the amylase level was normal. After the esophagogastroduodenoscopy, the patient underwent an uneventful laparoscopic distal pancreatectomy with splenectomy. Intraoperative ultrasonography showed a cystic

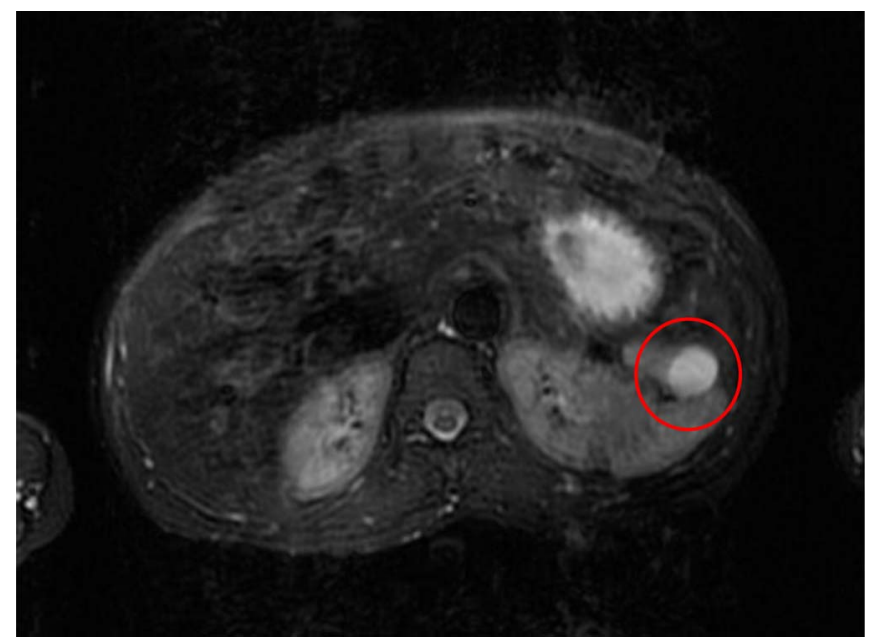

Figure 2. Magnetic resonance imaging scan of pancreatic tail mass (red circle).

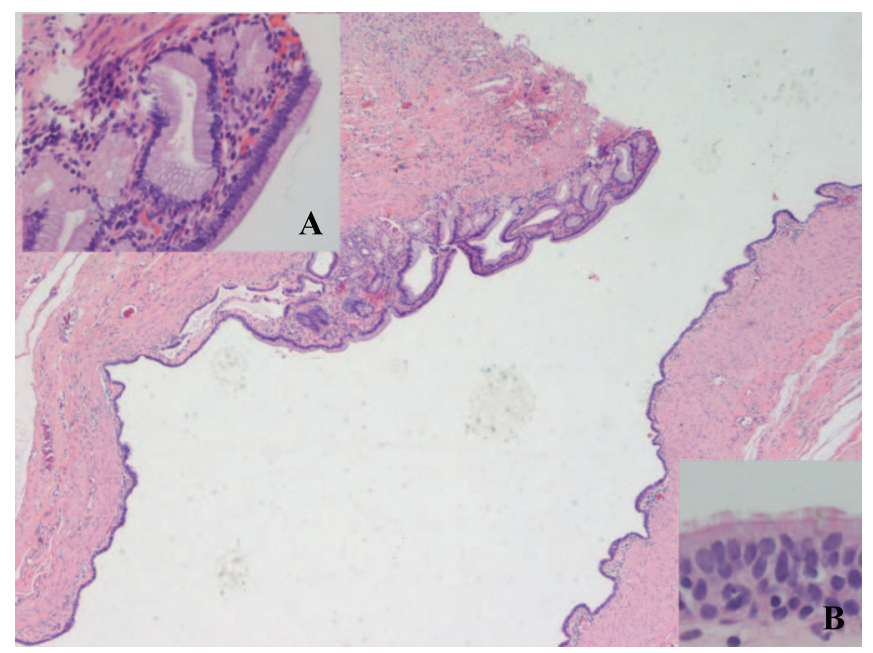

Figure 3. Composite image of enterogenous cyst histology (H\&E stain, magnification $40 \times$ ). Inset A: gastric mucosa (H\&E stain, magnification $100 \times$ ); Inset B: respiratory epithelium (H\&E stain, magnification $400 \times$ ).

lesion in the pancreatic tail within the hilum of the spleen. The fine-needle aspiration (FNA) results suggested a benign pathologic course, but at the time of surgery, an enterogenous cyst was low on the differential. Not only is it a rare lesion, but also pathologic analysis suggested an endometrial origin rather than an enteric duplication cyst. The distal location and intimacy with the hilum of the spleen made enucleation challenging, requiring a splenectomy with resection of the pancreatic tail. No gross intraoperative images were taken. The pathologic findings of the specimen described an entirely benign cyst measuring $2.0 \times 1.5 \times 1.2$ $\mathrm{cm}$, composed of mucosal, submucosal, and muscular layers 
with a lumen lined with rare foci of gastric mucosa (Figure 3) and ciliated respiratory-type epithelium. The spleen and lymph nodes were normal. The patient had an uneventful postoperative course.

\section{DISCUSSION}

Enterogenous cysts of the foregut are defined as those "arising from the intestines" and are lined with epithelium that simulates gastric or normal intestinal tissue. ${ }^{3}$ Those that arise outside of the liver are exceedingly rare and represent both a diagnostic and therapeutic challenge. ${ }^{6,7}$ Recent reports have provided a tool for the classification of these lesions, but there is still no consensus on the management. ${ }^{3}$ Ruling out a malignant process, such as mucinous cystic neoplasms (MCNs) and intraductal papillary mucinous neoplasms (IPMNs), is imperative. Because of the rarity of these lesions, there is little understanding of their biological activity and no precedent on the best treatment. With suspicious histologic elements, such as an elevated CEA level and the presence of mucin, as well as indeterminate imaging findings, resection becomes the standard of care. Resection could, in the face of this unknown natural history, be considered the more "conservative" form of treatment, eliminating any threat of malignant transformation.

Enterogenous cysts are difficult to differentiate from other cystic lesions on imaging. Because MCNs and IPMNs have malignant potential, resection for cystic lesions is often recommended. ${ }^{8}$ Where enterogenous cysts differ from other cystic neoplasms is in the pathology. In our patient, the EUS aspirate identified ciliated columnar cells and histiocytes consistent with a respiratory or bronchial origin. Elevated CEA levels in the cyst are consistent with respiratory-type epithelium. 9 This pathologic condition concurs with other case reports on enterogenous cysts. 5,10 Although this pathology is pathognomonic of enterogenous cysts, the FNA does not always provide sufficient samples of the cyst wall or architecture. ${ }^{11}$ Definitive histologic analysis in this case included gastric tissue and a surrounding rim of normal pancreatic tissue. These elements were not mentioned in the FNA sample. Obtaining the rim of normal pancreatic tissue in this distal lesion was the decisive factor for the surgical approach. Enucleation of the lesion could have been considered, given that it is acceptable for most benign lesions, if the lesion was more proximal, less involved with the splenic hilum, and less suggestive of an MCN. Surgical excision was necessary to confirm the suspicion that this was a benign process and rule out any malignant elements consistent with an MCN or IPMN.
As previously mentioned, the rarity of these pancreatic enterogenous cysts means that we do not fully understand their malignant potential. ${ }^{6}$ Hepatic foregut cysts, which are more common than those of the pancreas, have been noted to undergo malignant conversion. ${ }^{12,13}$ Also reported was the development of a malignancy arising from ciliated respiratory epithelium in a mature ovarian teratoma. ${ }^{14}$ Conversely, other reports suggested that these are developmental abnormalities and lack evidence of metaplasia. ${ }^{15}$ Such findings could argue that these benign developmental entities do not require surgery; however, in light of limited experience with enterogenous cysts, other models for the resection of benign processes should be considered. Surgery is recommended when there is an inability to monitor neoplastic change in benign or developmental processes. For example, choledochal cysts, lobular carcinomas in situ, or cryptogenic testes are all resected. Normal glands are resected for increased genetic risk as well (eg, thyroid in multiple endocrine neoplasia type $2 \mathrm{~B}$ ). Alternatively, there is often a mass effect with large cystic lesions, in addition to the inability to rule out the presence of a tumor, making resection the standard. ${ }^{16}$ The rare enterogenous cysts of the pancreas could be grouped among these benign processes for which surgery should be considered. Histologic findings on FNA may indeed indicate a benign process, but with the evidence of similar cysts undergoing dysplastic changes, a conservative approach involves surgical resection. This eliminates the concern over malignant transformation and any premalignant elements missed on FNA. Finally, resection provides a conclusive diagnosis and management for both the patient and the physician alike.

\section{CONCLUSION}

Enterogenous cysts of the pancreas are still a rare entity. Diagnostic challenges regarding this benign lesion include its similarities to malignant or premalignant cystic neoplasms that require excision for appropriate management. Because pathology is the definitive identifier of the final nature of these lesions, surgery is recommended as the conservative approach, especially in healthy patients with accessible lesions.

\section{References:}

1. De Oya JC, Puente JL, Villanueva A, et al. Enterogenous cyst of the ampulla of Vater. Digestion. 1969;2(4):201-208.

2. Dua KS, Vijayapal AS, Kengis J, Shidham VB. Ciliated foregut cysts of the pancreas: preoperative diagnosis using endoscopic 
ultrasound guided fine needle aspiration cytology-a case report with a review of the literature. Cytojournal. 2009;6:22.

3. del Carmen Gomez Mateo M, Munoz Forner E, Sabater Orti L, Ferrandez Izquierdo A. Foregut cystic malformations in the pancreas. Are definitions clearly established? J Pancreas. 2011; 12(4):420-424.

4. Woon CS, Pambuccian SE, Lai R, Jessurun J, Gulbahce HE. Ciliated foregut cyst of pancreas: cytologic findings on endoscopic ultrasound-guided fine-needle aspiration. Diagn Cytopathol. 2007;35:433-438.

5. Pins MR, Compton CC, Southern JF, Rattner DW, Lewandrowski KB. Ciliated enteric duplication cyst presenting as a pancreatic cystic neoplasm: report of a case with cyst fluid analysis. Clin Chem. 1992;38:1501-1503.

6. Muraoka A, Watanabe N, Ikeda Y, et al. Ciliated foregut cyst of the gallbladder: report of a case. Surg Today. 2003;33:718721.

7. Giakoustidis A, Morrison D, Thillainayagam A, et al. Ciliated foregut cyst of the gallbladder. A diagnostic challenge and management quandary. J Gastrointestin Liver Dis. 2014;23(2):207-210.

8. Tanaka M, Fernandez del Castillo C, Adsay V, et al. International consensus guideline 2012 for the management of IPMN and MCN of the pancreas. Pancreatology. 2012;13(1):183-197.

9. Nouwen EJ, Pollet DE, Eerdekens MW, Hendrix PG, Briers TW, De Broe ME. Immunohistochemical localization of placental alkaline phosphatase, carcinoembryonic antigen, and cancer antigen 125 in normal and neoplastic human lung. Cancer Res. 1986;46(2):866-876.
10. Idress MT, Reid-Nicholson M, Unger P, Jaffer S. Subhepatic ciliated foregut cyst. Ann Diagn Pathol. 2005;9:54-56.

11. Kluger MD, Tayar C, Belli A, et al. A foregut cystic neoplasm with diagnostic and therapeutic similarities to mucinous cystic neoplasms of the pancreas. J Pancreas. 2013; 14(4):446-449.

12. Zhang X, Wang Z, Dong Y. Squamous cell carcinoma arising in a ciliated hepatic foregut cyst: case report and literature review. Pathol Res Pract. 2009;205:498-501.

13. Furlanetto A, Dei Tos AP. Squamous cell carcinoma arising in a ciliated hepatic foregut cyst. Virchows Arch. 2002;441:296-298.

14. Sumi T, Ishiko O, Maeda K, Haba T, Wakasa K, Ogita S. Adenocarcinoma arising from respiratory ciliated epithelium in mature ovarian cystic teratoma. Arch Gynecol Obstet. 2002; 267(2):107-109.

15. Munshi IA, Parra-Davila E, Casillas VJ, Sleeman D, Levi JU. Ciliated foregut cyst of the pancreas. HPB Surg. 1998;11:117-119.

16. Liang MK, Yee HT, Song JW, Marks JL. Subdiaphragmatic bronchogenic cysts: a comprehensive review of the literature. Am Surg. 2005;71(12):1034-1041. 